\title{
POR QUE AS NARRATIVAS NACIONAIS PERMANECEM? REVISÃO DE LITERATURA SOBRE NOVAS PERSPECTIVAS NA PESQUISA DOS LIVROS DIDÁTICOS DE HISTÓRIA ${ }^{1}$
}

\author{
Maria Grever ${ }^{2}$ \\ Tina van der Vlies ${ }^{3}$
}

\begin{abstract}
Resumo: As narrativas nacionais servem frequentemente para mobilizar as massas para a guerra através de mitos e interpretações distorcidas do passado, enquanto as guerras funcionam como importantes fontes para a formação das narrativas nacionais. Como a história nacional normalmente permanece central no ensino de história, ainda que hoje de formas diversas de como se tratava o tema há 50 anos, é necessário compreender melhor estruturas e mecanismos subjacentes dessas narrativas nos livros didáticos de história. $\mathrm{O}$ presente artigo de revisão pretende delinear a interconexão histórica entre o nascimento dos estados nacionais e o ensino de história, assim como explicar a complexidade do livro didático de história como recurso educacional, para então identificar alguns problemas e desafios atuais para as pesquisas sobre os livros didáticos dessa disciplina. Evidenciaremos tendências promissoras das pesquisas, sobre narrativas nacionais conduzidas principalmente através da análise de imagens, da utilização de recursos digitais, e dos estudos sobre a autonomia da narrativa dos livros didáticos e da história dos livros didáticos em relação a outros meios de transmissão de conhecimento. Outro avanço recente na pesquisa dos livros didáticos é pensá-los de maneira holística. Estamos nos referindo aos estudos que consideram o livro didático como um todo: estrutura, periodização, intertextualidade das imagens e capítulos que, a princípio, parecem não estar centrados em uma história nacional. Estes estudos têm oferecido novas visões e explicações para a permanência das narrativas nacionais nos livros didáticos de história.
\end{abstract}

Palavras-chave: Livro Didático de História. Didática. Narrativas Nacionais. Análises Holísticas. Ensino de História.

\footnotetext{
${ }^{1}$ Tradução do original "Why national narratives are perpetuated: A literature review on new insights from history textbook research" por Catia Bulhosa Black e revisão técnica de Renata Siuda e Helenice Rocha. Este é um artigo de Open Acess distribuído nos termos da Creative Commons Attribution License, que permite uso, distribuição e reprodução irrestritos em qualquer meio, desde que o autor original e fonte sejam creditadas. Agradecemos às autoras, Maria Grever e Tina van der Vlies, bem como aos seus editores, pela simpatia à sua publicação neste dossiê da revista Escritas do Tempo. $\mathrm{O}$ artigo foi publicado originalmente em número especial da revista London Review of Education, cujo tema foi 'Negotiating the nation: Young people, national narratives and history education'. V. London Review of Education. Vol. 15, Number 2, July 2017. Nota dos editores: para essa tradução mantivemos as referências no formato do original.

${ }^{2}$ Maria Grever é professora de teoria e metodologia da história e diretora fundadora do Centro de Cultura Histórica da Universidade de Erasmus, em Roterdã (Holanda). Seus interesses de pesquisa envolvem: consciência histórica, teoria e historiografia, memória coletiva e identidade. Atualmente, lidera o projeto de pesquisa "Guerra! Cultura Popular e Herança Histórica Europeia de Grandes Conflitos Armados". É autora e organizadora de diversos livros e artigos científicos em revistas como Paedagogica Historica, o British Journal of Educational Studies, e o Journal of Curriculum Studies. Ver https://www.eur.nl/people/maria-grever

3 Tina van der Vlied é doutoranda e professora do Centro de Cultura Histórica da Universidade de Erasmus, em Roterdã (Holanda). Sua pesquisa sobre narrativas nacionais em livros didáticos de história ingleses e holandeses (1920-2010) tem o financiamento da Organização Holandesa de Pesquisa Científica (OHPS). Publicou recentemente o artigo "Narrativas multidirecionais das guerras nos livros didáticos de história", na revista Paedagogica Historica 52: 3 (2016). Este artigo é baseado em um trabalho que recebeu o Prêmio ISCHE de Início de Carreira, em 2015. Ver https://www.eur.nl/people/tina-van-dervlies.
}

Revista Escritas do Tempo - v. 2, n. 5, jul-out/2020 - p. 39-65 


\title{
WHY NATIONAL NARRATIVES ARE PERPETUATED: A LITERATURE REVIEW ON NEW INSIGHTS FROM HISTORY TEXTBOOK RESEARCH
}

\begin{abstract}
National narratives have often served to mobilize the masses for war by providing myths and distorted interpretations of the past, while conversely wars were major sources for producing national narratives. Because national history is very likely to remain a central topic in history education, albeit in ways that differ from how the topic was used fifty years ago, it is important to gain a greater understanding of the underlying structures and mechanisms of these narratives in history textbooks. After outlining the historical interconnectedness of the emerging nation states and history teaching, this review article explains the complexity of the history textbook as an educational resource. Next, we identify some current problems and challenges in history textbook research. We continue by discussing promising research trends related mainly to national narratives, such as the analysis of images, the use of digital tools, and studies of the autonomy of textbook narratives and of history textbooks in relation to other media. Another recent reorientation is textbook research that uses a holistic approach. By this we mean studies that examine the history textbook as a whole: composition, periodization, visual intertextuality and chapters that do not at first glance appear to focus on national history. These studies offer new insights and explanations for the perpetuation of national narratives in history textbooks.

Keywords: History Education. History Textbooks. Holistic Approach. National Narratives. Textbook Research.

\section{POURQUOI LES RECITS NATIONAUX RESTENT-ILS? UNE REVUE DE LITTÉRATURE SUR DE NOUVELLES PERSPECTIVE DAN LA RECHERCHE DE MANUELS SCOLAIRES D'HISTOIRE}

Résumé: Les récits nationaux servent souvent à mobiliser les masses pour la guerre à travers des mythes et des interprétations déformés du passé, tandis que les guerres fonctionnent comme des sources importantes pour la formation de récits nationaux. L'histoire nationale reste au cour de l'enseignement de l'histoire, bien qu'aujourd'hui différemment que le thème était pensé il y a 50 ans, il est nécessaire de mieux comprendre les structures et les mécanismes sous-jacents de ces récits dans les manuels d'histoire. Cet article vise à décrire l'interconnexion historique entre la naissance des États nationaux et l'enseignement de l'histoire, ainsi qu'à expliquer la complexité du manuel d'histoire en tant que ressource pédagogique, pour ensuite identifier certains problèmes et défis actuels pour la recherche sur les manuels de cette la discipline. Nous présenterons des tendances de recherche prometteuses, sur les récits nationaux conduits principalement par l'analyse d'images, l'utilisation des ressources numériques, et des études sur l'autonomie du récit et l'histoire des manuels scolaires par rapport à d'autres moyens de transmission des connaissances. Une autre avancée récente dans la recherche de manuels scolaires consiste à y penser de manière globale. Nous nous référençons à des études qui considèrent le manuel dans son intégralité: structure, périodisation, intertextualité des images et des chapitres qui, à première vue, ne semblent pas centrés sur une histoire nationale. Ces études ont offert de nouvelles perspectives et explications sur la permanence des récits nationaux dans les manuels scolaires d'histoire.

Mots-clés: Manuel scolaire d'histoire. Didactique. Récits nationaux. Analyse holistique. Enseignement de l'histoire.

\section{¿POR QUÉ LAS NARRATIVAS NACIONALES PERMANECEN? REVISIÓN DE LITERATURA SOBRE NOVAS PERSPECTIVAS EN LA PESQUISA DE LOS LIVROS DIDÁCTICOS DE HISTORIA}

\section{Resumen}

Comúnmente, las narrativas nacionales sirven para movilizar a las masas para la guerra por medio de mitos e interpretaciones distorsionadas del pasado, mientras las guerras funcionan como importantes fuentes para la formación das narrativas nacionales. Normalmente, la Historia 
nacional permanece central en la enseñanza de Historia. Sin embargo, hoy día ese tema es abordado de formas diversas de cómo se lo han tratado desde hace 50 años. Así, es necesario mejor comprender las estructuras y los mecanismos subyacentes de esas narrativas en los libros didácticos de Historia. Este artículo de revisión pretende delinear la interconexión histórica del nacimiento de los estados nacionales y de la enseñanza de Historia, explicar la complexidad del libro didáctico de Historia como un recurso educacional y, entonces, identificar algunos problemas y desafíos actuales referentes a las pesquisas sobre los libros didácticos de esa disciplina. Además, buscamos evidenciar las promisoras tendencias de pesquisas que tratan de narrativas nacionales, principalmente conducidas por análisis de imágenes, utilización de recursos digitales y estudios sobre la autonomía de la narrativa de los libros didácticos y sobre la historia de esos libros con relación a otros medios de transmisión de conocimiento. Un reciente avance en la pesquisa acerca de los libros didácticos es pensarlos de manera holística. Nos referimos a los estudios que consideran el libro didáctico como un conjunto de estructura, periodización, intertextualidad de imágenes y capítulos que, a principio, parecen no estar centrados em una Historia nacional. Esos estudios han ofrecido nuevas maneras de ver y explicar la permanencia de las narrativas nacionales en los libros didácticos de Historia.

Palabras clave: Libro Didáctico de Historia. Didáctica. Narrativas Nacionales. Análisis Holísticas. Enseñanza de Historia.

\section{Introdução}

No século XIX, a formação dos Estados Nacionais reforçou a profissionalização da história como disciplina científica. Apesar de estarem próximas do Estado, as historiografias nacionais se apresentaram como uma disciplina imparcial, fundada sobre a análise crítica de uma grande quantidade de fontes. Ao mesmo tempo, historiadores se dedicaram de forma incansável a construir uma infraestrutura institucional particular para a transmissão de valores patrióticos e de conhecimento histórico sobre a nação. Também influenciaram a consolidação do ensino de história e a produção dos livros didáticos de história. De acordo com Berger e Lorenz (2008: 12), "as elites de Estados e a maioria dos historiadores profissionais pressupuseram que a educação sobre uma história (nacional) era essencial para 'formação da nação' e para 'cidadania responsável'”.

No período de formação da nação, quando frequentemente se excluiu minorias étnicas e culturais (STUURMAN, 2007), tanto a história como ciência quanto aquela ensinada nas escolas foram as mais importantes construtoras de narrativas nacionais (WERTSCH, 2004). No novo milênio, tem-se observado em muitos países um forte renascimento de narrativas nacionais na educação. Dentre as queixas mais comuns nos debates públicos está a falta de familiaridade dos jovens com a história de seu país de residência. Jornais nacionais, programas de televisão e campanhas de internet têm acusado a história ensinada nas escolas de ser fragmentária, de apresentar narrativas relativistas e de marginalizar a história nacional (MOREAU, 2003; GREVER and STUURMAN, 2007; HAYDN, 2011). Os responsáveis pelas políticas públicas esperam 
um currículo de ensino de história dividido de forma cronológica e com uma narrativa nacional coerente e uniforme; os governos nacionais demandam a transmissão de cânones históricos que reforcem a identidade nacional (PHILLIPS, 2000). Entretanto, requisitos ligados a políticas identitárias são frequentemente incompatíveis com os critérios da história como profissão, por exemplo, o distanciamento e o juízo crítico (RÜSEN, 1994; VANSLEDRIGHT, 2008; GREVER et al., 2012; CHAPMAN e WILSCHUT, 2015). Em resumo, tanto a história como disciplina como aquela ensinada nas escolas estão profundamente interligadas aos processos de formação da identidade nacional.

A maioria dos pesquisadores de livros didáticos de história estão cientes dessa relação. Repoussi e Tutiaux- Guillon (2010), Foster (2011), Fuchs (2011), Hasberg (2012), entre outros especialistas, têm publicado artigos de revisão da literatura específica e dos avanços mais recentes na pesquisa dos livros didáticos de história. Para além de questões didáticas, métodos de pesquisa, produção, distribuição e recepção dos livros didáticos por estudantes e professores, têm se notado a preocupação com a pesquisa de temas controversos e de revisões dos livros didáticos que seguem mudanças nos regimes políticos. Em publicação recente, Fuchs e Sammler (2016, p. 12) propuseram que "uma reflexão crítica e sistemática sobre a história das revisões dos livros didáticos é um campo importante para a pesquisa sobre os livros didáticos", que transcende as fronteiras da Europa e dos Estados Unidos, englobando o Leste Asiático, a América Latina e a África.

O presente artigo tem como objetivo contribuir para as pesquisas sobre o livro didático de história de uma maneira especial. Nos dedicaremos a refletir principalmente sobre os livros didáticos de história que apresentam narrativas nacionais. Consideramos narrativas nacionais aquelas histórias (frequentemente canonizadas) que pensam a origem da nação e suas façanhas, bem como caracterizam as comunidades nacionais, e que são produzidas com o objetivo de dar um sentido ao passado e fazê-lo coeso com o presente e com um projeto de futuro (YADGAR, 2002; AMIN, 2014: 418; LÉTOURNEAU, 2017). Os enredos dessas narrativas são capitaneados pelas nações; são elas que se colocam como atores principais: declaram guerras, sofrem derrotas e triunfam (GREVER, 2007, p. 35). As narrativas nacionais alcançam com especial força os jovens e têm gerado consequências extremamente explosivas desde o século XIX. Elas frequentemente contribuíram para a mobilização das massas para a guerra e para que se cometessem genocídios a partir de mitos tendenciosos e distorções sobre o 
entendimento do passado (BERGER, 2007, p. 65), da mesma forma que as guerras geravam, em contrapartida, convincentes narrativas nacionais. A história nacional, ainda que bastante diferente do que se produzia há 50 anos, tende a permanecer como tópico central na história da educação, a despeito de tentativas de se implementar o ensino de outras abordagens culturais e geográficas (POPP, 2009; CARRETERO et al., 2012). Assim, faz-se importante questionar a perpetuação das narrativas nacionais nos livros didáticos para uma maior compreensão das estruturas e mecanismos que subjazem a esse tipo de narrativa (VAN DER VLIES, 2016, 2017). Além disso, analisar os livros didáticos “sob as lentes da 'nação' é uma forma eficiente de desvelar" conflitos sobre o conteúdo, propósitos, etc (MOREAU, 2003, p. 18).

A seguir, esclareceremos a identidade do livro didático como um recurso educacional. Depois, identificaremos alguns problemas e desafios na história da pesquisa sobre livros didáticos. Discutiremos, ao final, algumas tendências promissoras no campo da narrativa nacional, tais como a análise de imagens, o uso de ferramentas digitais e dos estudos sobre a autonomia da narrativa dos livros didáticos e da história dos livros didáticos em relação a outros meios de transmissão de conhecimento. Daremos especial atenção a uma nova forma de pesquisa sobre livro didático baseada na abordagem holística. Esses estudos pensam os livros didáticos como um todo: sua composição, periodização, intertextualidade das imagens e o papel dos capítulos que, a princípio, parecem não se concentrar na história nacional. A abordagem holística mostra que a organização do conhecimento histórico ampara a perpetuação das narrativas nacionais nos livros didáticos.

\section{Livros Didáticos de História: Um recurso pedagógico complexo}

O que exatamente queremos dizer com "livro didático" e "livro didático de história"? Issitt (2004, p. 685) propõe que os livros didáticos "são uma categoria muito confusa porque refletem uma multiplicidade de significados e usos", e "é justamente em razão dessa definição problemática que eles são fontes tão ricas". Sem dúvida, a tarefa de definir o que é livro didático é difícil. Muitas definições se concentram apenas em um aspecto, como por exemplo, seu propósito, uso ou status; outras definições são demasiado abrangentes (LEBRUN et al., 2002). De qualquer maneira, faz-se importante manter em mente que os livros didáticos fazem parte de um contexto mais amplo de educação, de política, de mídias, de cultura (popular) e de comércio, mas também de 
uma disciplina acadêmica específica, com seu status cultural próprio, sua tradição e jargão.

Os livros didáticos de história são recursos educacionais relacionados a disciplina histórica, produzidos com o objetivo de auxiliar ou — dependendo do país determinar os conteúdos do ensino formal de história e de sua aprendizagem, majoritariamente em escolas. Porque os livros didáticos são escritos com o objetivo de ensinar e de aprender, eles contêm perspectivas didáticas e pedagógicas implícitas ou explícitas. Consequentemente, os livros didáticos de história constituem um objeto bastante híbrido de pesquisa. Desde os anos 1980, eles se dividem majoritariamente em 3 categorias: 1) O livro didático principal com histórias, fragmentos de fontes, imagens, gráficos, mapas e referências de filmes e sites da internet; 2) O livro de exercícios, com diversas tarefas para os estudantes, também incluindo com frequência imagens, gráficos e mapas; 3) O Guia (livro) do Professor com as explicações sobre os temas históricos, referências a museus e outras mídias, recomendações didáticas e ajuda pedagógica (LEBRUN et al., 2002; HASBERG, 2012). ${ }^{4}$ Recentemente, os livros didáticos de história têm se tornado ainda mais híbridos, uma vez que passaram a incluir também conteúdos a serem acessados em sites da internet e outras mídias digitais (HAYDN, 2011; HAYDN e RIBBENS, 2017).

Como fontes primárias, os livros didáticos de história são de difícil trato para a pesquisa. Já de início, o livro didático precisa narrar eventos do passado de tal forma que os estudantes consigam compreender seus conteúdos, o que resulta inevitavelmente na seleção de determinados temas e em certa simplificação, dependendo da série na escola. Além do mais, os livros didáticos de história usufruem de um status especial: eles contêm conhecimentos históricos tidos como necessários para todos, com aprendizes e leitores possuindo um status epistemológico subordinado (ISSITT, 2004, p. 689). Tal realidade é confirmada pela sensação de autoridade que o livro didático de história habitualmente transmite (WINEBURG, 2001), reforçada por sua função canônica de ser um repositório da "verdade" e do conhecimento "válido" (OLSON, 1980, p. 194). Esse conhecimento histórico é selecionado e transmitido de uma geração para a outra: "Os livros didáticos de história preservam e transmitem verdades culturais de maneira intergeracional" (PORAT, 2001, p. 51). Um elemento que dá suporte a esse status especial é a forma como as palavras e as frases parecem objetivas e impessoais;

\footnotetext{
${ }^{4}$ Nota da revisora brasileira: No Brasil os livros didáticos de História habitualmente são constituídos por essas três categorias em um único volume impresso.
} 
histórias são contadas por um narrador onisciente. Na visão do público em geral, inclusive o de estudantes, os livros didáticos de história são considerados uma "fonte transcendental" de conhecimento (OLSON, 1980, p. 192), que comumente ocultam escolhas específicas e vieses ideológicos. Lowenthal (1998, p. 116) se refere a um diretor de um museu que reclamou que os jovens tinham aprendido na escola uma história como uma matéria finita e com respostas definitivas de certo ou errado. "A maioria dos textos históricos são escritos como se seus autores não existissem, como se fossem apenas instrumentos de uma inteligência divina, transcrevendo verdades oficiais".

Ademais, os livros didáticos transmitem valores, normas, comportamentos e ideologias escolhidas (ISSITT, 2004; PINGEL, 2008; LÄSSIG, 2009). Elie Podeh, que pesquisou livros didáticos israelenses, descreve-os como "outro braço do Estado, agentes de memória", e um tipo de "suprema corte histórica" porque "decidiam" o que era adequado de se incluir (PODEH, 2000, p. 66; APPLE e CHRISTIAN-SMITH, 1991). Por esta ótica, os livros didáticos de história são agentes coletivos de memória da nação (KAMMEN, 1993; LOWENTHAL, 1998). Funcionam como instrumentos de socialização e de política de identidade (LÄSSIG e POHL, 2009; BRAND, 2014).

Esses elementos que constituem o status especial dos livros didáticos de história provavelmente também estimulam o que chamamos de "guerras dos livros didáticos" nos anos 1990 e nos anos 2000 (ANDERSON, 1996; MACINTYRE e CLARK, 2003; GREVER e STUURMAN, 2007). Elites políticas, formadores de opinião, historiadores, especialistas em educação e professores negociam ou brigam sobre quais tópicos da história são relevantes e dignos de aparecerem (e de que maneira) nos livros didáticos. Isso se manifesta de maneira mais evidente em momentos de grandes transformações sociais e políticas, que geralmente deixam sua marca nos conteúdos e nas perspectivas dos livros didáticos de história. Um exemplo que ilustra bem esse processo é a mudança de regime na Rússia pós-1989 e a "imensa responsabilidade de revisar e reescrever os livros didáticos para se ajustarem à nova realidade" (KOROSTELINA, 2014, p. 297), que provocou acirrados debates em mídias de massa e fóruns profissionais sobre os conteúdos dos livros didáticos. Recentemente, o presidente russo Putin pediu a historiadores e historiadoras que desenvolvessem um "currículo de história que resultasse em uma história única, livre de "contradições internas e ambiguidades"" (KOVALYOVA, 2013). Esses projetos de produzir novos livros didáticos de história têm suscitado apreensão entre críticos que temem que a versão proposta de história seja 
"altamente politizada (ideologizada) e cheia de distorções grosseiras dos fatos" (KOVALYOVA, 2013, s/p). Outro caso é o do Conselho de Educação do Texas nos Estados Unidos, que desencadeou um acalorado debate sobre seus projetos de mudar os conteúdos dos livros didáticos de história das escolas em 2010. Historiadores e historiadoras protestaram contra as mudanças uma vez que sua parte significativa era historicamente incorreta e parecia motivada politicamente ('RON', 2013). Outros exemplos podem ser encontrados no Japão (SAALER, 2005; BUKH, 2007), Austrália (MACINTYRE AND CLARK, 2003), França (TUTIAUX-GUILLON, 2012), Grécia (REPOUSSI, 2006) e Israel (PORAT, 2001).

Contudo, como nos mostrou Barton (2011) e Foster (2011), é necessário muito cuidado para não equiparar diferentes contextos pedagógicos e arranjos curriculares que levem a formas equivocadas de se entender diferentes contextos nacionais e práticas de educação em história. Na Holanda, por exemplo, o governo central não se ocupa dos livros didáticos de História. Desde os anos 1980 e 1990, muitos livros didáticos de história holandeses passaram a incluir a construção crítica do conhecimento histórico em resposta aos debates que se davam entre especialistas em ensino de história e as revisões do currículo dessa disciplina. Consequentemente, esses livros didáticos não são "projetos de memória coletiva" que transmitem uma ideologia nacional específica. Já no caso da França, Alemanha, Estados Unidos e Japão, onde os governos (nacionais) controlam o currículo de história e os livros didáticos, a situação é bastante distinta (SYMCOX e WILSCHUT, 2009; SELDEN e NOZAKI, 2009; VAN BOXTEL e GREVER, 2011; VAN BERKEL, 2017). Outro caso é o dos professores e professoras do equivalente ao Ensino Médio no Reino Unido, que frequentemente utilizam seus próprios recursos para suplementar ou mesmo substituir livros didáticos de história (De BRUIJN, 2014, p. 25). William Marsden (2001, p. 55) evidenciou até uma tendência contra o livro didático na educação britânica, em razão das restrições financeiras que impedem seu uso enquanto as novas tecnologias têm proporcionado novos métodos de ensino. Terry Haydn (2011, p. 86) foi enfático ao pontuar que a situação do Reino Unido é provavelmente atípica em termos do uso do livro didático. Finalmente, podem também haver diferenças expressivas entre os ensinos nos níveis primários e secundários da educação escolar. É provável que professores do ensino fundamental utilizem o livro didático mais frequentemente. Em uma recente pesquisa na Finlândia, por exemplo, mostrou-se que a maioria dos professores e professoras de história e 
educação cívica atuando na educação básica usam o livro didático "sempre" ou "quase sempre" em sala de aula (SAKKI, 2014, p. 45).

Porque os livros didáticos de história são veículos de diferentes projetos sociais e políticos, e diferem em sua função em variados contextos nacionais e internacionais com demandas sociais e políticas distintas, a pesquisa sobre os livros didáticos encontra muitos mais desafios do que se costuma pensar (FOSTER, 2011, p. 5).

\section{O campo da pesquisa sobre o livro didático de história: Problemas e desafios}

A pesquisa sobre o livro didático tem uma forte tradição internacional. No início dos anos 1920, a Liga das Nações estimulou a pesquisa comparativa dos livros didáticos no tocante a estereótipos e como se tratava o "Outro" a fim de tentar um entendimento internacional. Depois da Segunda Guerra Mundial, a UNESCO e o Conselho Europeu continuaram esse tipo de pesquisa (PINGEL, 1999; NICHOLLS, 2003; FUCHS, 2011, p. 18). Desde sua fundação em 1951, o Instituto Georg Eckert (IGE), atualmente chamado de Instituto Georg Eckert para a Pesquisa Internacional Sobre o Livro Didático, na cidade alemã de Braunschweig, tem contribuído imensamente para a pesquisa sobre livros didáticos. Gradualmente, a pesquisa desenvolveu uma abordagem de questões específicas, contribuindo para uma análise crítica do contexto de produção, perspectivas de conteúdo e discursos nos livros didáticos. Esses estudos têm levantado questões, por exemplo, sobre as relações entre poder, ideologia e conhecimento histórico (FOSTER, 2011). Organizações internacionais têm apoiado pesquisas sobre livros didáticos de diferentes disciplinas escolares (história, geografia, estudos de paz, educação, estudos de mídia, sociologia e psicologia), mas as pesquisas sobre os livros didáticos de história ainda são consideradas cruciais para o campo geral da pesquisa sobre livros didáticos (KOROSTELINA, 2013).

A pesquisa sobre o livro didático de história pode ser considerada como historiografia educacional (DE KEYSER, 1998, p. 331) ou historiografia da escola (REPOUSSI e TUTIAUX-GUILLON, 2010, p. 154). Esse campo de pesquisa se concentra nos debates sobre a história ensinada na escola, a revolução didática, o envolvimento dos governos nacionais na construção do currículo de história, e do papel do mercado editorial. Apesar de estas pesquisas oferecerem considerações fascinantes sobre o substantivo desenvolvimento da história como disciplina e sua infraestrutura internacional, elas raramente aparecem nos compêndios de historiografia e filosofia da história. Uma das razões que pode explicar essa realidade é o fato de que, 
tradicionalmente, os estudos sobre os livros didáticos têm se concentrado na descrição das rupturas e permanências da representação histórica dos temas. A grande questão nessa área de pesquisa tende a ser: Que pessoas, eventos ou processos são marginalizados ou negligenciados? Como são apresentados? Em relação a essas questões faltam ainda metodologias genéricas. Em 1999, o antigo diretor do IGE, Falk Pingel, buscou enfrentar esse problema publicando um guia metodológico para a pesquisa sobre livros didáticos. Quatro anos depois, Nicholls pontuou que os métodos ainda eram "raramente discutidos com clareza ou profundidade", uma questão que permaneceu "como uma grande fraqueza no campo" da pesquisa sobre livros didáticos (NICHOLLS, 2003, p. 25). Outra provável razão para o pouco interesse historiográfico em relação às pesquisas sobre o livro didático é a questão de que o livro didático de história impresso tem sido considerado um recurso desatualizado. Pesquisar livros didáticos na era das digital humanities ${ }^{5}$ é uma tarefa antiquada, tanto no tocante ao objeto, quanto no tocante aos métodos. Ainda assim, Yet Repoussi e Tutiaux-Guillon (2010, p. 156) sugerem que os livros didáticos ainda são muito utilizados nas salas de aula, apesar de frequentemente combinados com sites da internet, aplicativos e outras mídias digitais.

Essa falta de interesse historiográfico é particularmente interessante porque os debates atuais entre historiadores e formadores de opinião no tocante ao ensino de história podem enriquecer muito com a abordagem longitudinal das pesquisas sobre livros didáticos de história. O que é dito nesses debates está frequentemente errado, nostálgico ou apresentado a partir de dicotomias simplistas, tais como "tradicional" (boa) versus "novo" ou "mau" ensino de História. A perspectiva histórica "tem o potencial de desvelar - e descartar - essas dicotomias" (VERSCHAFFEL e WILS, 2012, p. 4). Além disso, o conteúdo dos livros didáticos de história é frequentemente medido através dos critérios da historiografia acadêmica (VAN DER VLIES, 2014). Historiadores e historiadoras criticam a falta de determinados tópicos que eles/elas valorizam e detestam capítulos dedicados a temas "da moda", desaprovam a ênfase na construção crítica do conhecimento histórico, enquanto glorificam os livros de história escritos antes dos anos 1970 (SYMCOX AND WILSCHUT, 2009; VAN BOXTEL AND GREVER, 2011). Que história deve ser ensinada é uma questão que contrapõe historiadores com autores de livros didáticos há muito tempo. Um exemplo ilustrativo

\footnotetext{
${ }^{5}$ Nota da tradutora: Digital humanities refere-se a uma área de pesquisa, mantida em inglês pelo CNPq. Dessa forma, optamos por não traduzir o termo no texto, mas significa 'humanidades digitais'.
} 
desta questão são os debates extremamente politizados que se deram em 2011, no Reino Unido, quando se discutia o papel da história como disciplina no novo currículo nacional, com diversos historiadores renomados, como Simon Schama, tornando suas críticas públicas (VASAGAR, 2011). Mas o que os muitos críticos acadêmicos rapidamente esquecem - ou nem compreendem — são as consequências do contexto pedagógico. Os livros didáticos de história apresentam, dependendo da idade dos estudantes, versões simplificadas de processos muito complexos e multifatoriais que precisam ser ajustados a narrativas que sejam compreensíveis para os jovens. Não se quer dizer com isso que historiadores e historiadoras, bem como outros interlocutores, não possam estar certos em suas críticas. Em todos esses casos, entretanto, a forma repressora como se enfrentam essas discussões raramente contribui para se entender como e porque determinados tópicos históricos nos livros didáticos permanecem importantes para os autores e autoras, que se apegam a eles como se fossem "velhos cânones" (VAN DER VLIES, 2017, 2019).

Enquanto os historiadores e historiadoras do mundo acadêmico tendem a não reconhecer o valor da pesquisa historiográfica sobre os livros didáticos, o mesmo não acontece nos fóruns de formação de opinião pública, como mencionado. A pesquisa sobre livros didáticos causa até reações controversas, como ficou evidente quando a obra de James Loewen's 'Mentiras que meu professor me contou: Todos os erros do seu livro didático de história dos Estados Unidos' (Lies my Teacher Told Me: Everything your American history textbook got wrong, 1995) foi publicada. O sociólogo, David Horowitz, em particular, acusou a obra de não ser um "trabalho acadêmico" e sim "uma polêmica sectária contra o ensino tradicional da história estadunidense e contra o que o autor entende como um registro negro do passado americano" (History News Network, 2007).

O livro de Loewen — um best-seller premiado — foi baseado em pesquisa feita com 12 livros didáticos de história amplamente adotados nas escolas dos Estados Unidos, em observações feitas em salas de aula e entrevistas com estudantes e professores do equivalente ao Ensino Médio no país. O autor não apenas desvelou aspectos mitológicos, equivocados e eurocêntricos nos livros didáticos, como também evidenciou temas frequentemente ignorados, como a exploração americana do trabalho escravo negro. Assim, o autor demonstrou como a história estadunidense nos livros didáticos favorece uma versão positiva do papel dos brancos na construção nacional. 
Para além do debate sobre quem está certo, a polêmica entre Loewen e Horowitz claramente nos mostra alguns dos desafios do campo da pesquisa sobre livros didáticos.

Primeiramente, a pesquisa sobre as representações históricas nos livros didáticos tende a revelar o que NÃO aparece neles e o que foi distorcido ou censurado. Para além do exemplo do livro acima mencionado de Loewen, podemos citar alguns capítulos do volume 'Censurando a História' (Censoring History, Hein e Selden, 2000). Esse tipo de pesquisa demonstra que os livros didáticos frequentemente perpetuam "velhas narrativas" e contribuem para uma amnesia estrutural, entre outras formas de "esquecimento" nas sociedades, como, por exemplo, causadas pelas intervenções estatais: quando os governos demandam que sejam feitas revisões nos livros didáticos ou impõem sua ideia do que é o conhecimento "certo". Tópicos polêmicos do passado são omitidos, ignorados ou apagados (CONNERTON, 2008). Entretanto, esses estudos não esclarecem muito sobre as razões por trás da perpetuação de determinadas narrativas nos livros didáticos de história, como acontece, por exemplo, em sociedades onde o governo não determina os conteúdos deles. Portanto, junto à questão das deficiências dos livros didáticos de história, uma análise aprofundada sobre as estruturas internas dos livros didáticos se faz necessária. Ao invés de se evidenciar o que falta, pesquisadores e pesquisadoras de livros didáticos têm se preocupado muito mais com que está neles e por quê.

Um segundo desafio que precisa ser enfrentado é que precisamos de mais pesquisas sobre o impacto (nacional) das narrativas dos livros didáticos de história para o entendimento do passado por parte de professores e estudantes. Para além do estudo de Loewen, existem outros exemplos: Fournier e Wineburg (1997) pediram para que crianças entre 10 e 12 anos fizessem desenhos de três tipos de figuras históricas depois da leitura de pequenos textos. As impressões dos pesquisadores foram de que os desenhos das crianças tinham sido influenciados pelo que tinham aprendido e visto nos livros didáticos tradicionais adotados nas escolas. Também se investigou a relação entre a idade das crianças e sua compreensão histórica: a identificação com narrativas nacionais comuns em livros didáticos era constante (CARRETERO e VAN ALPHEN, 2014). Outros pesquisadores analisaram a seleção que estudantes faziam das narrativas nacionais quando concluíam o equivalente ao ensino médio nos Estados Unidos, considerando as múltiplas perspectivas nas construções narrativas dos professores (KROPMAN et al., 2015). 
Um terceiro desafio é a questão da autoria. É preciso saber mais sobre quem de fato escreve os livros didáticos de história. Os autores e autoras são autônomos e formados em história? Em caso negativo, o que isso diz sobre a qualidade dos livros didáticos de história? Em diversos países, como nos Estados Unidos, a produção de livros didáticos de história é altamente influenciada por interesses comerciais das editoras (MOREAU, 2003), que contratam autores anônimos (ghost writers) e celebram acordos com historiadores e historiadoras profissionais de renome que assinam as publicações. A realidade na Alemanha e na Holanda é completamente diferente. Em sua tese de doutoramento, Van Berkel (2017) mostrou que nestes países a maioria dos autores e autoras de livros didáticos eram formados em história, ainda que esse seja mais o caso da Alemanha em comparação à Holanda. As diversas formas de autoria no campo influenciam não apenas o conteúdo histórico e a qualidade das tarefas contidas nos livros didáticos, como também podem ajudar a explicar algumas das práticas de duplicação de narrativas "velhas" ou familiares.

Na próxima secção, discutiremos algumas das promissoras novas pesquisas que podem potencialmente esclarecer como e porque as narrativas, em particular as narrativas nacionais, são perpetuadas nos livros didáticos de história.

\section{Tendências promissoras na pesquisa sobre o livro didático e as narrativas nacionais}

Nas últimas décadas, a pesquisa sobre os livros didáticos de história tem mudado profundamente em relação aos seus métodos e teorias. Evoluiu da análise descritiva dos livros didáticos para abraçar o uso de métodos quantitativos e qualitativos provenientes das ciências sociais, da narratologia e dos estudos de mídia. As orientações para análises sistemáticas de livros didáticos têm sido aprimoradas. Já temos alguns estudos introdutórios e pioneiros nesta área (FOSTER E CRAWFORD, 2006; PIGEL, 2008; HAYDN, 2011; REPOUSSI e TUTIAUX-GUILLON, 2010; FUCHS 2011; FUCHS e SAMMLER, 2016).

Diversos pesquisadores têm desenvolvido novos recursos para a análise dos livros didáticos (MORGAN e HENNING, 2013). No mais, com base nos recentes avanços no campo das digital humanities, o Instituto Georg Eckert criou um departamento específico para aprofundar a investigação sobre novos métodos e recursos digitais específicos para cada disciplina: o Departamento de Informação Digital e Pesquisa de Infraestrutura (GEI, 2017). Esses recursos ligados à pesquisa sobre os livros 
didáticos têm propiciado novas oportunidades de coleta de dados e de rastreamento de padrões de palavras. Pesquisadores estão usando softwares como o Atlas, ti e Alcaste para transformar em código os conteúdos dos livros didáticos e assim poder examinar as características vocabulares de determinados discursos. Com esses recursos digitais, pesquisadores e pesquisadoras podem descobrir padrões narrativos no uso da retórica e de analogias. Como resultado, uma pesquisa comparativa de larga escala sobre narrativas nacionais nos livros didáticos de história tem dado indicativos sobre algumas características gerais dessas narrativas e seu papel em contextos específicos de formação nacional, bem como sobre a construção e a permanência dessas narrativas.

Um bom exemplo dessas questões é o interessante estudo de Sakki (2014). A pesquisadora se propôs a analisar como livros didáticos de educação cívica de cinco países europeus retrataram a integração da Europa. Sua metodologia incluiu as análises quantitativas e qualitativas sobre o que e como se escrevia da integração europeia e permitiu a conclusão de que a história da integração era contada a partir das perspectivas nacionais de cada um desses países. Em outro artigo, Sakki (2016) mostra que os livros didáticos franceses pensam a integração europeia como um processo significativo da memória compartilhada do pós-guerra, fazendo também referências a uma ideia antiga de Europa, enquanto os livros didáticos ingleses pensam a integração a partir de suas políticas internas. Ademais, o tipo de pesquisa feita por Sakki também mostra um deslocamento das narrativas nacionais em direção às narrativas pósnacionais.

Essa construção teórica não significa, porém, o fim das narrativas nacionais, mas coloca nações específicas nas fronteiras diferentes e mutáveis argumentando que as relações entre as pessoas e condições de pertencimento não podem ser compreendidas sem se fazer referência a um contexto mais amplo de mudanças geopolíticas (PEASE, 1997). Essa tendência também é visível no projeto da pesquisa desenvolvida pelo COST Action IS 1205, e apresentada em julho de 2016, que analisa representações históricas (COST Action IS 1205, 2016). Um grupo de trabalho, liderado por Tibor Polya e Eva Fülöp, inclui pesquisa sobre como a União Europeia é retratada nos livros didáticos de história.

Outra recente tendência é a influência das representações visual e espacial na pesquisa sobre os livros didáticos (FUCHS e SAMMLER, 2016, p. 11-12). De acordo com Pieter de Bruijn (2014), as representações visuais da história que progressivamente aparecem com mais frequência nos livros didáticos de história, como é o caso de objetos 
históricos famosos ou fontes históricas, entram nas edições com o propósito de ilustrar ou ensinar. Pesquisadores e pesquisadoras desenvolveram novas formas de análise iconográfica nos livros didáticos, uma vez que o uso de imagens tem aumentado tanto nos livros didáticos principais, como nos livros de exercícios para os estudantes. Em relação às narrativas nacionais, pesquisadores e pesquisadoras têm descoberto como a seleção de fotografias "icônicas" sustenta essas narrativas e como os autores e autoras contribuem para a perpetuação dessas imagens icônicas (KLEPPE, 2013).

Além do mais, a pesquisa comparativa entre mapas e imagens pode revelar como um mesmo evento histórico é retratado de maneiras diferentes nas diversas narrativas nacionais, dependendo da sua interpretação como triunfo ou derrota de uma nação, que constitui o tema principal. O estudo de Mario Carretero (2014), por exemplo, comparou a colonização da América e a representação de Colombo em livros didáticos mexicanos e espanhóis. Enquanto os livros espanhóis continham informações biográficas sobre Colombo e muitas imagens, os livros mexicanos mencionavam brevemente sua morte e dedicavam mais textos e imagens para a crueldade do tratamento dos indígenas e mexicanos. Nos livros didáticos de ambos os países, as informações textuais e iconográficas concorriam para o estímulo da imaginação histórica sobre a colonização da América.

Inspirados na narratologia, pesquisadores e pesquisadoras de livros didáticos de história têm também se dedicado a examinar processos menos visíveis, mas que são importantes para a explicação das rupturas e continuidades das narrativas nacionais nos livros didáticos (VAN DER VLIES, 2014, 2016). Depois de analisar alguns mitos fundadores dos Estados Unidos, Ray Raphael afirma que as narrativas dos livros didáticos conseguem "sobreviver" inclusive apesar de estudos científicos que relativizam ou apontam para erros, por causa de "três razões, intimamente interligadas: elas nos dão uma identidade coletiva, elas constituem boas histórias e, por fim, nós acreditamos que elas são patrióticas" (RAPHAEL 2004, p. 5). Como vimos, já há algum tempo pesquisadores de livros didáticos tem debatido as questões das políticas identitárias e dos valores patrióticos; contudo, a ênfase na autonomia das narrativas representa uma abordagem promissora para pesquisas futuras acerca da perpetuação das narrativas nacionais. Algumas narrativas nacionais são demasiado interessantes para não serem contadas: "O bem luta contra o mal, David derrota Golias, e os homens sábios triunfam sobre os tolos (...). Mesmo que não se conte histórias verdadeiras, elas 
alimentam a imaginação e funcionam como um fio condutor da narrativa" (RAPHAEL, 2004, p. 5); ver também Van der Vlies (2016).

Despontando de antigas tradições, a permanência de determinadas versões do passado se dá em razão de cumprirem bem o papel de cânon e de continuarem "relevantes para formações culturais posteriores" (OLICK e ROBBINS, 1998, p. 129; GREVER, 2007, p. 41). Portanto, determinadas versões só mudarão quando não "conseguirem mais dar conta do entendimento do presente ou quando perderem sua relevância para o presente" (OLICK e ROBBINS, 1998, p. 130). Daí os livros didáticos de história frequentemente repetem narrativas familiares em razão de fatores culturais. Para melhorar nosso entendimento deste processo e compreender as estruturas internas dos livros didáticos de história, precisamos analisá-los como um todo integrado, segundo uma outra tendência promissora de avanços no campo.

\section{Uma abordagem holística: A estrutura das narrativas nacionais}

As narrativas nacionais podem ser altamente padronizadas e constituídas de acordo com uma estrutura comum (FELDMAN, 2001). Esses padrões podem ser bastante preponderantes e persistentes, mesmo que os detalhes sobre determinada narrativa tenham mudado. Consequentemente, alguns estudiosos falam de narrativa nacional complexa, "construída a partir de um conjunto de narrativas secundárias, mitos, símbolos, metáforas e imagens" (YADGAR, 2002, p. 58). Para desnudar a complexidade dos estudos dos livros didáticos, precisamos de uma abordagem holística: com ênfase nos livros didáticos de história como um todo integrado. Em 2003, Thomas Höhne propôs novas questões de pesquisa com o objetivo de compreender melhor as narrativas dos livros didáticos e o tipo de conteúdo que apresentam. Höhne afirma que essas questões podem suplantar os critérios normativos tradicionais na pesquisa sobre livros didáticos, tais como "verdadeiro" e "falso", para dar lugar a uma análise integral do discurso do livro didático (HÖHNE, 2002, p. 13).

Narrativas específicas têm seus significados no contexto do livro didático como um todo, o que é, com certeza, aplicável às narrativas nacionais. Isso significa que a estrutura da narrativa e a composição do livro didático de história e suas narrativas específicas sobre eventos do passado estão intimamente ligadas. Mudanças na estrutura da narrativa do livro didático, como no caso de transformações sociais associadas a diferentes visões do passado, têm impacto sobre a função e o significado de toda a gama de narrativas (nacionais) existentes. Algumas narrativas específicas desaparecem, 
enquanto outras que eram "secundárias" são enfatizadas e estendidas. Um exemplo disso é o caso dos livros didáticos holandeses, que passaram a dar maior ênfase ao comércio transatlântico de escravos e à escravidão, que deixaram de ser temas "secundários" ligados aos empreendimentos comerciais do início da era moderna, ganhando recentemente maior visibilidade na narrativa nacional, apesar de ainda provocar críticas entre os especialistas (KLEIN, 2017, p. 76). Isso se deu em razão da persistência dos migrantes das antigas colônias holandesas do Suriname e Antilhas descendentes de escravos — que continuaram demandando a visibilidade do seu passado e do papel dos holandeses no mesmo (VAN STIPRIAAN, 2007).

Em sua atual pesquisa sobre as narrativas nacionais nos livros didáticos de história ingleses e holandeses, Van der Vlies analisa-os dessa maneira. Inspirando-se no conceito de Michael Rothberg de 'memória multidirecional', ela cunha a noção de 'narrativas multidirecionais dos livros didáticos': narrativas nos livros didáticos de história que combinam diferentes histórias, lugares e temporalidades de uma forma produtiva que gera significado das combinações históricas. Rothberg afirma que a memória coletiva é muitas vezes vista como disputa sem vencedores na qual as coisas estão ou presentes ou ausentes; daí sua postura de pensar a 'memória multidimensional' através das "transferências dinâmicas que acontecem entre diferentes lugares e tempos durante o ato de recordar" (ROTHBERG, 2009, p. 11). Van der Vlies (2016, 2019) demonstra essas transferências dinâmicas no contexto das narrativas dos livros didáticos: referências cruzadas e trocas entre narrativas apresentadas em diferentes versões nacionais do passado nos livros didáticos. Exemplo memorável de livros didáticos ingleses é a referência cruzada entre a Armada Espanhola de 1588 e a ameaça do Blitzkrieg alemão em 1940. Diferenças como o fato de que, em 1588, o perigo vinha do mar, enquanto em 1940 do céu, não enfraquecem a comparação, já que os dois eventos expressam a mesma coisa: o medo da invasão. As narrativas dos livros didáticos mostram padrões de interpretação nos quais podem ser inseridos diferentes dados e eventos. Desta forma, é possível detectar os padrões de significado nas narrativas da história (nacional). Os livros didáticos de história podem, portanto, conter um conjunto de narrativas que reverberam: juntas, elas constituem, afirmam e inculcam a narrativa nacional (VAN DER VLIES, 2017, 2019).

As transferências dinâmicas nas narrativas dos livros didáticos são reforçadas pelos usos de novas mídias. As narrativas dos livros didáticos estão sendo paulatinamente incorporadas em um mundo (re)mediado, especialmente na era dos 
livros didáticos digitais, que facilmente redirecionam os estudantes a acessar o YouTube ou outros sites da internet via hiperlinks.

Nessa perspectiva, Eleftherios Klerides (2010, p. 334) aponta para formas híbridas e dinâmicas de livros didáticos de história, que ele descreve como "multifacetados", uma "combinação de discursos" e um "campo interdiscursivo". Na mesma linha da pesquisa, Heinze (2010, p. 125) sugere que os livros didáticos de história não devem ser pensados como "espelhos" que refletem determinados discursos, mas como "mediadores de discurso, já que apresentam ímpetos didáticos e metodológicos que reproduzem esses discursos".

Uma abordagem holística dos livros didáticos de história também pode elucidar de que forma a organização do conhecimento histórico contribui para a perpetuação de narrativas nacionais. Eventos de temporalidades distintas podem ser inter-relacionados tendo como base um enredo comum ou uma vertente principal da narrativa (WERTSCH, 2004). Esta abordagem também pode nos ajudar a compreender melhor como se organiza o tempo cronológico (diacrônico e sincrônico), a seleção e os nomes dados aos eventos e períodos, bem como a adoção de conceitos coligatórios (por exemplo, o Renascimento, a Revolução Industrial, a Guerra Fria) que amarram diferentes elementos e narrativas em um mesmo enredo (JANSEN, 2010, p. 243; GREVER et al., 2012).

Ainda mais importante é que a abordagem holística transcenda a análise representacional limitada a uma pessoa, evento ou período, separados do contexto do livro como um todo. Bert Vanhulle (2009, p. 264), que se utilizou da abordagem holística em sua análise da "concepção da narrativa" nos livros didáticos de história belgas entre 1945-2004, propõe que “a ênfase se dá na estrutura e nas suas consequências para o texto como representação histórica, não na forma como são retratados eventos passados/ grupos/identidades, nas consequências do ensino ou consequências para as mentes dos jovens aprendizes e da sociedade em geral". Consequentemente, ele se interessa em localizar o início da narração. Se pergunta se a história tem uma fonte (fons), "um poço do qual saem as nascentes da história", e se esse é caso, para qual direção corre a água. Porque o pesquisador pensa o "todo" do livro didático de história como uma representação histórica, é possível identificar concepções veladas da história: interpretações específicas sobre a relação entre o passado, o presente e o futuro, como questão da decadência, do progresso e da 
escatologia (ADRIAANSEN, 2015; VAN DER VLIES, 2016; GREVER e ADRIAANSEN, 2017).

Alexander Albicher (2012, p. 43) seguiu a abordagem de Vanhulle ao considerar o livro didático de história como uma narrativa completa. Discutindo como os livros didáticos holandeses tratam o passado e o presente durante o período entre 1945-85, conseguiu formular uma série de perguntas: Os livros didáticos estão expondo majoritariamente eventos e contingências, ou processos e estruturas? Existe um "motor" que move a história? Para além da periodização e da categorização da história, também estudou as descontinuidades e os momentos de viradas/inflexões, que são, por exemplo, importantes para a composição de uma narrativa nacional já que marcam ao mesmo tempo o fim e um novo começo. Como Zerubavel (2003, p. 85) mostrou: "A descontinuidade temporal é uma forma de descontinuidade mental, e a forma como dividimos o passado é uma manifestação de como dividimos nosso espaço mental em geral". Os momentos de inflexão destacam as descontinuidades da experiência de vida das pessoas e servem para ancorar as comunidades mnemônicas (GREVER, 2001, p. 11, 18). Portanto, para compreender a perpetuação das narrativas nacionais nos livros didáticos de história, é necessário pensarmos neles também em relação a uma configuração cultural mnemônica mais ampla.

\section{Considerações finais}

No presente artigo, discutimos inovações nas pesquisas sobre os livros didáticos de história em relação à perpetuação das narrativas nacionais. A razão de nossa escolha se deu pelo fato de que as histórias nacionais provavelmente continuarão a ter muita importância no ensino de história. O que podemos concluir até o momento? Primeiramente, tendo em vista que os livros didáticos de história são recursos educacionais híbridos e são alvo de intensas controvérsias políticas, a pesquisa sobre esse objeto apresenta muitos mais problemas e desafios do que se antecipa. Compêndios e manuais de historiografia e filosofia da história, em especial, raramente dedicam qualquer atenção para a pesquisa sobre o livro didático de história. Identificamos, desta forma, três desafios: 1) As controvérsias sobre o ensino de história frequentemente se concentram no que não está nos livros didáticos, ou estão presentes de forma distorcida, o que torna mais difícil enfrentar a questão sobre o que está nos livros didáticos; 2) as pesquisas sobre o impacto dos livros didáticos de história na forma como professores e estudantes entendem o passado ainda são raras; e 3) é necessário saber mais sobre a 
autoria dos livros didáticos de história já que essa questão pode esclarecer muito as razões por trás da persistência de velhas narrativas.

Ademais, defendemos que os debates atuais entre historiadores e formadores de opinião sobre o ensino de História podem se beneficiar da abordagem longitudinal das pesquisas sobre o livro didático de história e de uma análise aprofundada de suas estruturas narrativas internas. Isso ajuda a evitar dicotomias simplistas e nostálgicas entre o "bem" e o "mal" no ensino de História. Também discutimos avanços promissores nas pesquisas sobre as narrativas nacionais. No tocante aos métodos, pontuamos que novas ferramentas para a análise dos livros didáticos estão sendo desenvolvidas e aplicadas, tais como softwares que codificam os conteúdos dos livros didáticos e examinam as características do vocabulário utilizado em determinado discurso. Reconhecendo que a iconografia se tornou parte considerável dos livros didáticos de história e dos livros de exercícios dos estudantes, pesquisadores e pesquisadoras têm explorado novas formas de analisar as imagens contidas nos livros: como, por exemplo, fotografias "icônicas" contribuem para as narrativas nacionais. Essas metodologias são importantes contribuições para o entendimento da perpetuação e da transformação das narrativas nacionais nos livros didáticos de história, tais como a descoberta de padrões nos livros didáticos que nos auxiliam a formular as características gerais das narrativas nacionais.

Por fim, uma tendência promissora na pesquisa sobre os livros didáticos de história é a abordagem holística: a análise do livro didático com um todo integrado. Nesses estudos, pesquisadores e pesquisadoras analisam as dinâmicas da estrutura interna e sua inter-relação com outras mídias. Essa abordagem revela como o conhecimento histórico é organizado, arranjado no tempo cronológico, como se dá a seleção de eventos e períodos e como se usam os conceitos coligatórios. A abordagem holística, além disso, permite que pesquisadores e pesquisadoras dos livros didáticos identifiquem concepções subjacentes à história: visões sobre a relação entre passado, presente e futuro. Essa tendência evidencia a construção das narrativas nacionais nos livros didáticos de história e de que formas (menos visíveis) são perpetuadas. Através dessa abordagem, podemos também compreender melhor as mudanças nas funções e sentidos das narrativas nacionais existentes nos livros didáticos de história, bem como seu potencial poder de mobilização. 


\section{Referências}

Adriaansen, R.-J. (2015) The Rhythm of Eternity: The German youth movement and the experience of the past ,1900-1933. New York: Berghahn Books.

Albicher, A. (2012) Heimwee naar het heden: Betrokkenheid en distantie in het Nederlandse geschiedenisonderwijs (1945-1985). PhD thesis, University of Leuven.

Amin, S.N. (2014) 'The impact of identity politics in challenging national narratives: A case study among Canadian Muslims'. Studies in Ethnicity and Nationalism, 14 (3), 418-35.

Anderson, M. (1996) Imposters in the Temple: A blueprint for improving higher education in America. Stanford, CA: Hoover Institution Press.

Apple, M.W. and Christian-Smith, L.K. (eds) (1991) The Politics of the Textbook. London: Routledge.

Barton, K.C. (2011) 'Wars and rumors of war: The rhetoric and reality of history education in the United States'. In Taylor, T. and Guyver, R. (eds) History Wars and the Classroom: Global perspectives. Charlotte, NC: Information Age Publishing, 187-202.

Berger, S. (2007) 'Writing national histories in Europe: Reflections on the pasts, presents, and futures of a tradition'. In Jarausch, K.H. and Lindenberger, T. (eds) Conflicted Memories: Europeanizing contemporary histories. New York: Berghahn Books, 55-68.

Berger, S. and Lorenz, C. (2008) 'Introduction: National history writing in Europe in a global age'. In Berger, S. and Lorenz, C. (eds) The Contested Nation: Ethnicity, class, religion and gender in national histories.

Basingstoke: Palgrave Macmillan, 1-23. Brand, L.A. (2014) Official Stories: Politics and national narratives in Egypt and Algeria. Stanford: Stanford University Press.

Bukh, A. (2007) 'Japan's history textbooks debate: National identity in narratives of victimhood and victimization'. Asian Survey, 47 (5), 683-704.

Carretero, M. (2014) 'History learning research in Spain and Latin America'. In Köster, M., Thünemann, H. and Zülsdorf-Kersting, M. (eds) Researching History Education: International perspectives and disciplinary traditions. Schwalbach: Wochenschau Verlag, 56-80.

Carretero, M., Asensio, M. and Rodríguez-Moneo, M. (eds) (2012) History Education and the Construction of National Identities. Charlotte, NC: Information Age Publishing.

Carretero, M. and van Alphen, F. (2014) 'Do master narratives change among high school students? A characterization of how national history is represented'. Cognition and Instruction, 32 (3), 290-312. 
Chapman, A. and Wilschut, A. (eds) (2015) Joined-Up History: New directions in history education research. Charlotte, NC: Information Age Publishing.

Connerton, P. (2008) 'Seven types of forgetting'. Memory Studies, 1 (1), 59-71. COST Action IS 1205 (2016) 'Agents through time: how do people "make history"? Final conference of COST Action IS 1205.' Online. http://media.wix.com/ugd/0e5c62_d155af6496fd4ca1b6f0288d6daa7e2e.pdf (accessed 24 April 2017).

De Bruijn, P. (2014) Bridges to the Past: Historical distance and multiperspectivity in English and Dutch heritage educational resources. PhD thesis, Erasmus University Rotterdam.

De Keyser, R. (1998) 'Schoolboekenhistoriografie: Een verkenning'. In Tollebeek, J., Verbeeck, G. and Verschaffel, T. (eds) De lectuur van het verleden: Opstellen over de geschiedenis van de geschiedschrijving aangeboden aan Reginald de Schryver. Leuven: Leuven University Press, 331-48.

Feldman, C.F. (2001) 'Narratives of national identity as group narratives: Patterns of interpretive cognition'. In Brockmeier, J. and Carbaugh, D. (eds) Narrative and Identity: Studies in autobiography, self and culture. Amsterdam: John Benjamins Publishing Company, 129-44.

Fournier, J.E. and Wineburg, S.S. (1997) 'Picturing the past: Gender differences in the depiction of historical figures'. American Journal of Education, 105 (2), 160-85.

Foster, S. (2011) 'Dominant traditions in international textbook research and revision'. Education Inquiry, 2 (1), 5-20.

Foster, S. J. and Crawford, K.A. (eds) (2006) What Shall We Tell the Children? International perspectives on school history textbooks. Charlotte, NC: Information Age Publishing.

Fuchs, E. (2011) 'Current trends in history and social studies textbook research'. Journal of International Cooperation in Education, 14 (2), 17-34.

Fuchs, E. and Sammler, S. (2016) Textbooks between Tradition and Innovation: A journey through the history of the Georg Eckert Institute. Braunschweig: Georg Eckert Institute.

GEI (Georg Eckert Institute) (2017) 'Digital information and research infrastructures'. Online. $\quad$ www.gei.de/en/departments/digital-information-and-researchinfrastructures.html (Accessed 12 April 2017).

Grever, M. (2001) De enscenering van de tijd. Inaugural address, Erasmus University Rotterdam, 14 December. Rotterdam: Overbosch.

(2007) 'Plurality, narrative and the historical canon'. In Grever, M. and Stuurman, S. (eds) Beyond the Canon: History for the twenty-first century. Basingstoke: Palgrave Macmillan, 31-47. 
Grever, M. and Adriaansen, R.-J. (2017) 'Historical culture: A concept revisited'. In Carretero, M., Berger, S. and Grever, M. (eds) Palgrave Handbook of Research in Historical Culture and Education. Basingstoke: Palgrave Macmillan, 73-89.

Grever, M., De Bruijn, P. and Van Boxtel, C. (2012) 'Negotiating historical distance: Or, how to deal with the past as a foreign country in heritage education'. Paedagogica Historica, 48 (6), 873-87.

Grever, M. and Stuurman, S. (eds) (2007) Beyond the Canon: History for the twentyfirst century. Basingstoke: Palgrave Macmillan.

Hasberg, W. (2012) 'Closed or broken narrations? Work-orders as elements of historical narrations in history textbooks'. In Erdmann, E., Cajani, L., Khodnev, A.S., Popp, S., Tutiaux-Guillon, N. and Wrangham, G.D. (eds) From Historical Research to School History: Problems, relations, challenges (Yearbook of the International Society for History Didactics). Schwalbach: Wochenschau Verlag, 125-44.

Haydn, T. (2011) 'The changing form and use of textbooks in the history classroom in the 21st century: A view from the UK'. In Erdmann, E., Cajani, L., Khodnev, A.S., Popp, S., Tutiaux-Guillon, N. and Wrangham, G.D. (eds) Analyzing Textbooks: Methodological issues (Yearbook of the International Society for History Didactics). Schwalbach: Wochenschau Verlag, 67-88.

Haydn, T. and Ribbens, K. (2017) 'Social media, new technologies and history education'. In Carretero, M., Berger, S. and Grever, M. (eds) Palgrave Handbook of Research in Historical Culture and Education. Basingstoke: Palgrave Macmillan (forthcoming).

Hein, L. and Selden, M. (eds) (2000) Censoring History: Citizenship and memory in Japan, Germany, and the United States. Armonk, NY: M.E. Sharpe.

Heinze, C. (2010) 'Historical textbook research: Textbooks in the context of the "grammar of schooling"'. Journal of Educational Media, Memory, and Society, 2 (2), $122-31$.

History News Network (2007) 'James Loewen: Criticized by David Horowitz'. 22 January. Online. http://historynewsnetwork.org/article/34458 (accessed 2 February 2017).

Höhne, T. (2003) Schulbuchwissen: Umrisse einer Wissens- und Medientheorie des Schulbuches. Frankfurt: Johann Wolfgang Goethe-Universität.

Issitt, J. (2004) 'Reflections on the study of textbooks'. History of Education, 33 (6), 683-96.

Jansen, H. (2010) Triptiek van de tijd: Geschiedenis in drievoud. Nijmegen: Vantilt. Kammen, M. (1993) Mystic Chords of Memory: The transformation of tradition in American culture. New York: Vintage Books. 
Klein, S. (2017) 'Preparing to teach a slavery past: History teachers and educators as navigators of historical distance'. Theory and Research in Social Education, 45 (1), 75109.

Korostelina, K.V. (2013) History Education in the Formation of Social Identity: Toward a culture of peace. Basingstoke: Palgrave Macmillan.

(2014) 'Legitimizing an authoritarian regime: Dynamics of history education in independent Russia'. In Williams, J.H. (ed.) (Re)Constructing Memory: School textbooks and the imagination of the nation. Rotterdam: Sense Publishers, 293-310.

Kovalyova, A. (2013) 'Is Vladimir Putin rewriting Russia's history books?'. NBC News, 28 November. Online. www.nbcnews.com/news/other/vladimir-putin-rewriting-russiashistory-books-f2D11669160 Accessed 2 February 2017).

Kleppe, M. (2013) Canonieke icoonfoto's: De rol van (pers)foto's in de Nederlandse geschiedschrijving. Delft: Eburon.

Klerides, E. (2010) 'Imagining the textbook: Textbooks as discourse and genre'. Journal of Educational Media, Memory, and Society, 2 (1), 31-54.

Kropman, M., Van Boxtel, C. and Van Drie, J. (2015) 'Small country, great ambitions: Prospective teachers' narratives and knowledge about Dutch history'. In Chapman, A. and Wilschut, A. (eds) Joined-Up History: New directions in history education research. Charlotte, NC: Information Age Publishers, 57-84.

Lässig, S. (2009) 'Textbooks and beyond: Educational media in context(s)'. Journal of Educational Media, Memory, and Society, 1 (1), 1-20.

Lässig, S. and Pohl, K.H. (2009) 'History textbooks and historical scholarship in Germany'. History Workshop Journal, 67, 125-39.

Lebrun, J., Lenoir, Y., Laforest, M., Larose, F., Roy, G.-R., Spallanzani, C. and Pearson, M. (2002) 'Past and current trends in the analysis of textbooks in a Quebec context'. Curriculum Inquiry, 32 (1), 51-83.

Létourneau, J. (2017) 'Teaching national history to young people today'. In Carretero, M., Berger, S. and Grever, M. (eds) Palgrave Handbook of Research in Historical Culture and Education. Basingstoke: Palgrave Macmillan, 227-42.

Loewen, J.W. (1995) Lies My Teacher Told Me: Everything your American history textbook got wrong. New York: New Press.

Lowenthal, D. (1998) The Heritage Crusade and the Spoils of History. Cambridge: Cambridge University Press.

Macintyre, S. and Clark, A. (2003) The History Wars. Melbourne: Melbourne University Press. 
Marsden, W.E. (2001) The School Textbook: Geography, history and social studies. London: Woburn Press.

Moreau, J. (2003) Schoolbook Nation: Conflicts over American history textbooks from the Civil War to the present. Ann Arbor: University of Michigan Press.

Morgan, K. and Henning, E. (2013) 'Designing a tool for history textbook analysis'. Forum: Qualitative Social Research, 14 (1), Article 7. Online. www.qualitativeresearch.net/index.php/fqs/article/view/1807/3460 (accessed 21 July 2016).

Nicholls, J. (2003) 'Methods in school textbook research'. International Journal of Historical Learning, Teaching and Research, 3 (2), 11-26.

Olick, J.K. and Robbins, J. (1998) 'Social memory studies: From "collective memory" to the historical sociology of mnemonic practices'. Annual Review of Sociology, 24, $105-40$.

Olson, D.R. (1980) 'On the language and authority of textbooks'. Journal of Communication, 30 (1), 186-96. Pease, D.E. (1997) 'National narratives, postnational narration'. Modern Fiction Studies, 43 (1), 1-23.

Phillips, R. (2000) 'Government policies, the state and the teaching of history'. In Arthur, J. and Phillips, R. (eds) Issues in History Teaching. London: RoutledgeFalmer, $10-23$.

Pingel, F. (1999) UNESCO Guidebook on Textbook Research and Textbook Revision. Hannover: Verlag Hahnsche Buchhandlung.

(2008) 'Can truth be negotiated? History textbook revision as a means to reconciliation'. Annals of the American Academy of Political and Social Science, 617 (1), 181-98.

Podeh, E. (2000) 'History and memory in the Israeli educational system: The portrayal of the Arab-Israeli conflict in history textbooks (1948-2000)'. History and Memory, 12 (1), 65-100.

Popp, S. (2009) 'National textbook controversies in a globalizing world'. In Erdmann, E., Cajani, L., Khodnev, A.S., Popp, S., Tutiaux-Guillon, N. and Wrangham, G.D. (eds) History Teaching in the Crossfire of Political Interests (Yearbook of the International Society for History Didactics, 2008/9). Schwalbach: Wochenschau Verlag, 109-22.

Porat, D. (2001) 'A contemporary past: History textbooks as sites of national memory'. In Dickinson, A.,

Raphael, R. (2004) Founding Myths: Stories that hide our patriotic past. New York: New Press.

Repoussi, M. and Tutiaux-Guillon, N. (2010) 'New trends in history textbook research: Issues and methodologies toward a school historiography'. Journal of Educational Media, Memory, and Society, 2 (1), 154-70. 
'Ron' (2013) 'Texas textbook controversy'. US Slave blog, 25 May. Online. http://usslave.blogspot. co.uk/2013/05/texas-textbook-controversy.html (accessed 2 February 2017).

Rothberg, M. (2009) Multidirectional Memory: Remembering the Holocaust in the age of decolonization. Stanford: Stanford University Press.

Rüsen, J. (1994) 'Was ist Geschichtskultur? Überlegungen zu einer neuen Art, über Geschichte nachzudenken'. In Rüsen, J. Historische Orientierung: Über die Arbeit des Geschichtsbewusstseins, sich in der Zeit zurechtzufinden. Köln: Böhlau, 211-34.

Saaler, S. (2005) Politics, Memory and Public Opinion: The history textbook controversy and Japanese society. Munich: Iudicium.

Sakki, I. (2014) 'Social representations of European integration as narrated by school textbooks in five European nations'. International Journal of Intercultural Relations, $43,35-47$.

(2016) 'Raising European citizens: Constructing European identities in French and English textbooks'. Journal of Social and Political Psychology, 4 (1), 444-72.

Selden, M. and Nozaki, Y. (2009) 'Japanese textbook controversies, nationalism, and historical memory: Intra- and inter-national conflicts'. The Asia-Pacific Journal, 7 (24), Article 5, 1-24.

Stuurman, S. (2007) 'Integration, assimilation and exclusion in the making of modern nations'. Tijdschrift voor Geschiedenis, 120, 446-59.

Symcox, L. and Wilschut, A. (eds) (2009) National History Standards: The problem of the canon and the future of teaching history. Charlotte, NC: Information Age Publishing.

Tutiaux-Guillon, N. (2012) 'A traditional frame for global history: The narrative of modernity in French secondary school'. In Carretero, M., Asensio, M. and RodriguezMoneo, M. (eds) History Education and the Construction of National Identities. Charlotte, NC: Information Age Publishing, 109-24.

Van Berkel, M. (2017) Plotlines of Victimhood: Holocaust education in German and Dutch history textbooks, 1960-2010. PhD thesis, Erasmus University Rotterdam (forthcoming).

Van Boxtel, C. and Grever, M. (2011) 'Between disenchantment and high expectations: History education in the Netherlands, 1968-2008'. In Erdmann, E. and Hasberg, W. (eds) Facing, Mapping, Bridging Diversity: Foundation of a European discourse on history education: Part 2. Schwalbach: Wochenschau Verlag, 83-116.

Van der Vlies, T. (2014) 'Geschiedverhalen en hun eigen dynamiek: Schoolboeken als zelfstandig genre'. Kleio, 55 (1), 30-5. 
Van der Vlies, T. (2016) 'Multidirectional war narratives in history textbooks'. Paedagogica Historica: International Journal of the History of Education, 52 (3), 30014.

Van der Vlies, T. (2017) 'Echoing national narratives in English history textbooks'. In Carretero, M., Berger, S. and Grever, M. (eds) Palgrave Handbook of Research in Historical Culture and Education. Basingstoke: Palgrave Macmillan, 243-58.

Van der Vlies, T. (2019) Echoing Events. The Perpetuation of National Narratives in English and Dutch history textbooks, 1920-2010. PhD thesis Erasmus University Rotterdam.

Vanhulle, B. (2009) 'The path of history: Narrative analysis of history textbooks: A case study of Belgian history textbooks (1945-2004)'. History of Education: Journal of the History of Education Society, 38 (2), 263-82.

VanSledright, B. (2008) 'Narratives of nation-state, historical knowledge, and school history education'. Review of Research in Education, 32 (1), 109-46.

Van Stipriaan, A. (2007) 'Disrupting the canon: The case of slavery'. In Grever, M. and Stuurman, S. (eds) Beyond the Canon: History for the twenty-fi rst century. Basingstoke: Palgrave Macmillan, 205-19.

Vasagar, J. (2011) 'History textbooks are "failing pupils"'. The Observer, 13 March. Online. Www.theguardian.com/education/2011/mar/13/schools-failing-at-teachinghistory (accessed 2 February 2017).

Verschaffel, T. and Wils, K. (2012) 'History education and the claims of society: An historical approach'. Journal of Educational Media, Memory, and Society, 4 (1), 1-6.

Wertsch, J.V. (2004) 'Specifi c narratives and schematic narrative templates'. In Seixas, P. (ed.) Theorizing Historical Consciousness. Toronto: University of Toronto Press, 4962.

Wineburg, S. (2001) Historical Thinking and Other Unnatural Acts: Charting the future of teaching the past. Philadelphia: Temple University Press.

Yadgar, Y. (2002) 'From the particularistic to the universalistic: National narratives in Israel's mainstream press, 1967-97'. Nations and Nationalism, 8 (1), 55-72.

Zerubavel, E. (2003) Time Maps: Collective memory and the social shape of the past. Chicago: University of Chicago Press.

Artigo recebido em 30 de junho de 2020. Aprovado em 04 de agosto de 2020. 